\title{
The previous stretching to resistance training promotes adaptations on the postsynaptic region in different myofiber types
}

\section{Carolina dos Santos Jacob}

São Paulo State University

Gabriela Klein Barbosa

São Paulo State University

Mariana Pasquini Rodrigues

São Paulo State University

Jurandyr Pimentel Neto

São Paulo State University

\section{Lara Caetano Rocha}

São Paulo State University

Adriano Polican Ciena ( $\nabla$ adriano.ciena@unesp.br)

São Paulo State University

\section{Research Article}

Keywords: neuromuscular junction, motor endplate, muscle hypertrophy, static stretching, resistance training.

Posted Date: April 28th, 2021

DOl: https://doi.org/10.21203/rs.3.rs-465730/v1

License: (c) (i) This work is licensed under a Creative Commons Attribution 4.0 International License. Read Full License 
The previous stretching to resistance training promotes adaptations on the postsynaptic region in different myofiber types

Carolina dos Santos Jacob, Gabriela Klein Barbosa, Mariana Pasquini Rodrigues, Jurandyr Pimentel Neto, Lara Caetano Rocha, *Adriano Polican Ciena

Laboratory of Morphology and Physical Activity (LAMAF), Institute of Biosciences (IB), São Paulo State University, Rio Claro, SP, Brazil.

\section{Corresponding Author:}

Adriano Polican Ciena, $\mathrm{PhD}$

Institute of Biosciences (IB), São Paulo State University.

Av 24A, n 1515, 13506-900, Rio Claro, SP, Brazil.

Tel. +55 $193526-4346$

ORCID: 0000-0002-2912-3376

e-mail: adriano.ciena@unesp.br 


\begin{abstract}
This investigation revealed the postsynaptic morphological adaptations in static stretching, resistance training, and their association in adult male Wistar rats. We processed the soleus and plantaris muscle for histochemical (muscle hypertrophy) and postsynaptic region imaging techniques. We observed muscle hypertrophy in both groups submitted to resistance training, even though the cross-section area is larger when there is no previous static stretching. The soleus postsynaptic region revealed an increase in compactness and fragmentation index in combined exercise. The resistance training promotes high adaptations in the postsynaptic area of plantaris; moreover, the previous static stretching decreased this area. In conclusion, the neuromuscular system's components respond according to the myofiber type even though it is the same physical exercise. Besides, static stretching (isolated or combined) plays a crucial role in neuromuscular adaptations.
\end{abstract}

Keywords: neuromuscular junction, motor endplate, muscle hypertrophy, static stretching, resistance training. 


\section{Introduction}

Exercise training promotes increased neuromuscular activity, and demonstes the plasticity of the neuromuscular junction (NMJ), resulting in improved endurance and resistance training in different ages ${ }^{1,2}$. Moreover, the NMJ presented a high potential in postsynaptic components adaptations. The increase in acetylcholine receptors (AChR) and endplate compactness, ensuring efficient communication between the pre- and postsynaptic region, and prolonged muscle fatigue $\mathrm{e}^{3,4,5}$.

Resistance training (RT) has been used to promote chronic muscular adaptations in humans and demonstrated to be a principal modality for increasing muscle strength, endurance, power, hypertrophy, and motor performance ${ }^{6,7}$. These adaptations are predominantly due to increasing the muscle cross-section area through the differentiation and proliferation of satellite cells by the release of inflammatory cytokines and growth factors $^{8}$. Also, RT is a method where manipulating their variables (series, repetitions, frequency, overload, and rest interval) is directly proportional to the progress of adaptive biological responses ${ }^{9}$.

Static stretching has been highly widespread among individuals of all physical activity levels, commonly prescribed during the warm-up of strength training, conditioning, and rehabilitation ${ }^{10,11}$. Also, static stretching does not require much time or effort, and has a low risk of injury in young adults, and has shown beneficial postural stability results ${ }^{12,13}$.

Evidence suggests that stretching performed before resistance training can directly influence strength production, reduce the number of repetitions, the total volume of training, and muscle hypertrophy ${ }^{14,15,16}$.

It is essential to understand the possible effects in the postsynaptic region of the NMJ caused by static stretching and resistance training, especially in their association. Therefore, we investigated the neuromuscular junction plasticity in 8 weeks of static stretching, resistance training and your association in adult male Wistar rats. 


\section{Results}

\section{Cross-section area (CSA)}

In the histochemical analysis, the Type I and II CSAs from the soleus muscle and the Type I, IIa, and IIx CSAs from plantaris of all experimental groups were obtained (Table 1).

Soleus: the CSA of Types I $(p<0.05)$ and II fibers $(p<0.0001)$ of soleus of the ST group were smaller compared to NT. The CSA of Type I fibers $(\mathrm{p}<0.0001)$ of RT was larger compared to NT. Additionally, the CSA of Type II fibers $(\mathrm{p}<0.01)$ of RT was smaller than that found in NT.

The CSA of Types I $(p<0.05)$ and II $(\mathrm{p}<0.001)$ fibers of SRT were larger than NT group. The CSA of Types I $(\mathrm{p}=0.0760)$ and II $(\mathrm{p}<0.0001)$ fibers of SRT were larger than ST. Besides, the CSA of Type I ( $p<0.0001)$ fibers of SRT were smaller, and the Type II $(\mathrm{p}<0.0001)$ fibers were larger than that found in the RT group.

Plantaris: the CSA of Types I, IIa $(\mathrm{p}<0.0001)$, and IIx $(\mathrm{p}<0.001)$ fibers of plantaris of ST were both larger compared to NT. The CSA of Types I ( $p<0.0001)$, IIa $(\mathrm{p}<0.0001)$, and IIx $(\mathrm{p}<0.0001)$ fibers of RT were both larger compared to NT. Furthermore, the CSA of Types I $(p<0.0001)$ and IIa $(p<0.0001)$ fibers of SRT were larger, and the Type IIx ( $p>0.9999)$ fibers were smaller than that found in NT.

In the SRT Group, the CSA of Types I $(\mathrm{p}<0.0001)$ and IIa $(\mathrm{p}<0,01)$ fibers were larger, and Type IIx $(\mathrm{p}<0.0001)$ fibers were smaller compared to ST. Additionally, the CSA of Types IIa $(p<0.001)$ and IIx $(p<0.0001)$ fibers of SRT were both smaller, and the Type $\mathrm{I}(\mathrm{p}<0.0001)$ fibers were larger than that found in RT. 
Table 1: Mean \pm standard deviation of cross-section area $\left(\mu \mathrm{m}^{2}\right)$ of myofibers Type I and II in the soleus muscle and I, IIa, IIx in the plantaris muscle of the No-Training (NT), Resistance Training (RT), Stretching Training (ST), and Stretching-Resistance Training (SRT) groups.

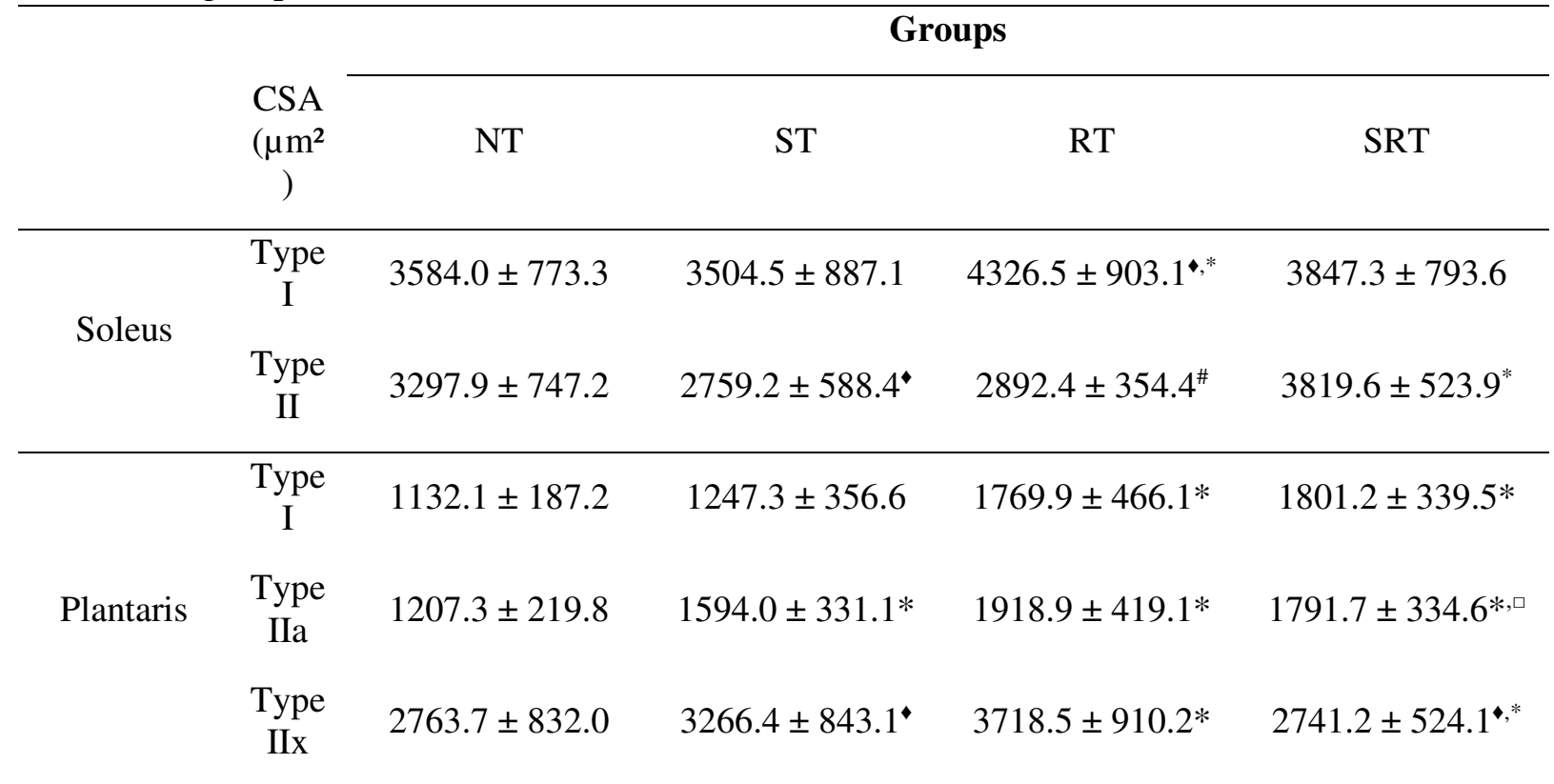

Soleus: Type I: ${ }^{\star} \mathrm{NT} \neq \mathrm{RT}(\mathrm{p}<0.001),{ }^{*} \mathrm{ST} \neq \mathrm{RT}(\mathrm{p}<0.0001)$, and ${ }^{*} \mathrm{SRT} \neq \mathrm{RT}(\mathrm{p}<0.0001)$; Type II: ${ }^{\star} \mathrm{NT} \neq \mathrm{ST}(\mathrm{p}<0.001),{ }^{*} \mathrm{NT} \neq \mathrm{RT}(\mathrm{p}<0.05),{ }^{*} \mathrm{NT} \neq \mathrm{SRT}(\mathrm{p}<0.0001)$, and ${ }^{*} \mathrm{RT} \neq \mathrm{SRT}$ $(\mathrm{p}<0.0001)$. Plantaris: Type I: $* \mathrm{NT} \neq \mathrm{RT} \quad(\mathrm{p}<0.0001), * \mathrm{NT} \neq \mathrm{SRT}(\mathrm{p}<0.0001)$, and $* \mathrm{ST} \neq \mathrm{SRT}(\mathrm{p}<0.0001)$. Type IIa: $* \mathrm{NT} \neq \mathrm{ST}(\mathrm{p}<0.0001),{ }^{*} \mathrm{NT} \neq \mathrm{RT}(\mathrm{p}<0.0001),{ }^{*} \mathrm{NT} \neq \mathrm{SRT}$ $(\mathrm{p}<0.0001)$, and ${ }^{\square} \mathrm{ST} \neq \mathrm{SRT} \quad(\mathrm{p}<0.01)$. Type IIx: $* \mathrm{NT} \neq \mathrm{RT} \quad(\mathrm{p}<0.0001), \quad{ }^{\star N T} \neq \mathrm{ST}$ $(\mathrm{p}<0.001), * \mathrm{RT} \neq \mathrm{SRT}(\mathrm{p}<0.0001)$, and ${ }^{\star S T} \neq \mathrm{SRT}(\mathrm{p}<0.001)$.

\section{Postsynaptic regions}

The area and perimeter of the endplate and AChR of soleus presented smaller in the ST group than NT. Moreover, in the ST group, the diameter $(p=0,2652)$, compactness $(\mathrm{p}=0.1384)$, and number of $\mathrm{AChR}$ clusters $(\mathrm{p}>0.9999)$ of the endplate were smaller than that found in the NT group. Additionally, the NT and ST groups presented an endplate fragmentation index of 0.71 and 0.55 , respectively (Figure 1 ).

The area and perimeter of the endplate and AChR of soleus were both smaller in the RT group compared to NT. Furthermore, in the RT group, the diameter $(>0.9999)$ and compactness $(\mathrm{p}<0.3316)$ of the endplate were smaller; and the number of AChR clusters ( $p>0.9999)$ was higher than that found in the NT. The RT presented an endplate fragmentation index of 0.64 (Figure 1).

The area and perimeter of the endplate and AChR of soleus were both smaller in the SRT group compared to NT ( $\mathrm{p}=0.8010)$. Besides, the endplate diameter and the 
number of AChR clusters of SRT were both smaller compared to NT ( $p>0.9999)$. In SRT, the compactness endplate showed larger than NT ( $>$ 0.9999). The SRT presented an endplate fragmentation index of 0.59 (Figure 1).

The area and perimeter of the endplate of SRT of soleus were smaller compared to ST. However, the AChR area and perimeter, and endplate compactness were larger in SRT than that found in the ST group. Furthermore, the endplate diameter ( $p>0.9999)$ and the number of AChR clusters ( $\mathrm{p}>0.9999)$ of SRT showed minor value compared to ST (Figure 1).

The area of the endplate $(\mathrm{p}=0.6070)$ and AChR $(\mathrm{p}>0.9999)$ of SRT was smaller than RT. The endplate perimeter ( $p>0.9999)$ and diameter $(\mathrm{p}>0.9999)$ of SRT were both smaller than that found in the RT. Besides, the AChR perimeter ( $>0.9999)$, the endplate compactness $(p=0.2340)$, and the number of AChR clusters $(p>0.9999)$ of SRT were larger than RT (Figure 1).
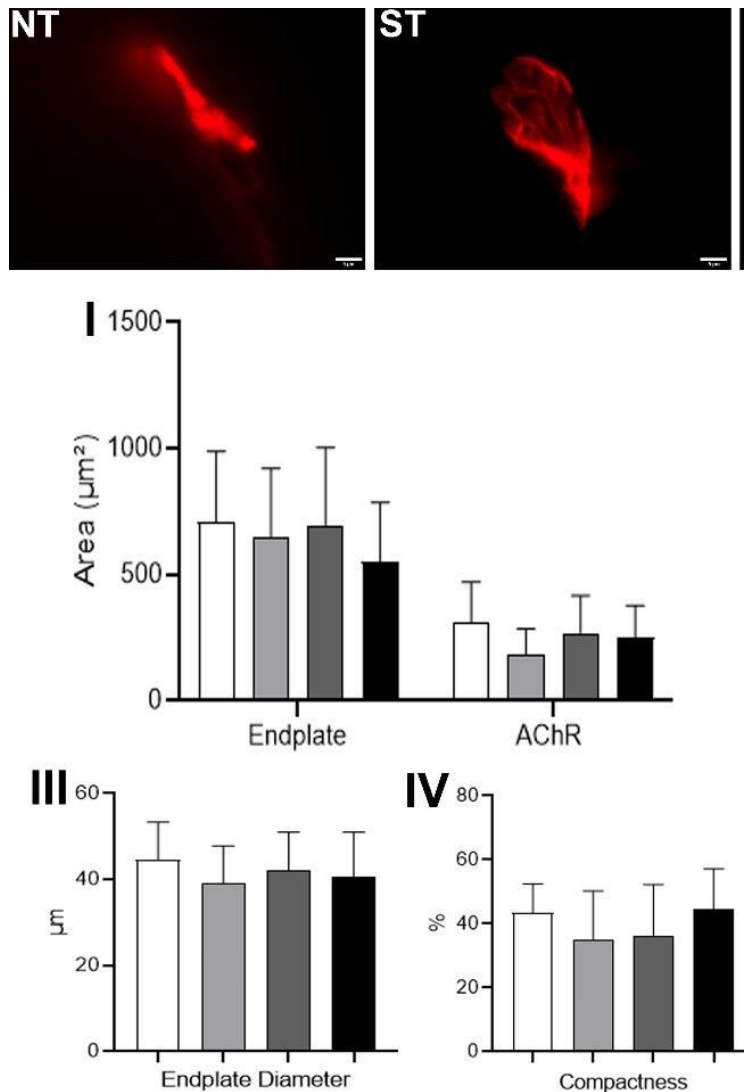

$\square \mathrm{NT} \quad \square \mathrm{ST}$
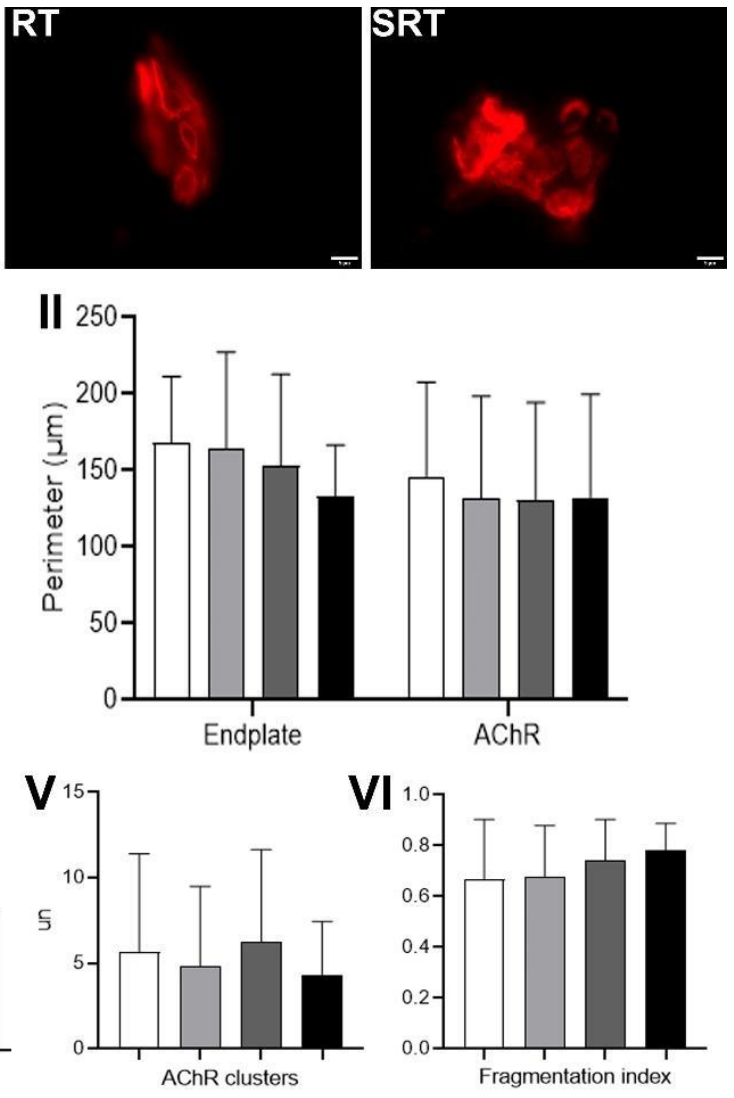

$\square$ RT

- SRT

Figure 1: Postsynaptic AChR region of the soleus muscle of No-Training (NT), Stretching Training (ST), Resistance Training (RT), and Stretching-Resistance Training (SRT) groups. Bars: $5 \mu \mathrm{m}$. (I) Mean \pm standard deviation values of the endplate and AChR areas $\left(\mu \mathrm{m}^{2}\right)$. (II) Mean \pm standard deviation values of the endplate and AChR perimeters $(\mu \mathrm{m})$. (III) Mean \pm standard deviation values of endplate diameter $(\mu \mathrm{m})$. (IV) 
Mean \pm standard deviation values of endplate compactness (\%). (V) Mean \pm standard deviation values of the number of AchR clusters (un). (VI) Mean \pm standard deviation values of the fragmentation index.

The AChR area and perimeter, and the area and perimeter of the endplate of plantaris were larger $(\mathrm{p}<0.01)$ in the ST group than in the NT. The endplate diameter ( $p>0.9999)$, compactness ( $>00.9999)$, and the number of AChR clusters $(p=0.0381)$ of ST were larger than those found in the NT. Additionally, the NT and ST groups presented an endplate fragmentation index of 0.66 and 0.73 , respectively (Figure 2).

The AChR area and perimeter, and the area and perimeter of the endplate were larger $(\mathrm{p}<0.05)$ in RT than in the NT. Furthermore, the endplate diameter $(\mathrm{p}>0.9999)$, compactness ( $p>0.9999)$, and the number of AChR clusters $(\mathrm{p}<0.01)$ of RT were both larger than NT. The RT presented an endplate fragmentation index of 0.74 (Figure 2).

The endplate area of SRT of plantaris was smaller compared to NT ( $\mathrm{p}=0.997)$. Additionally, the endplate perimeter ( $p>0.9999)$, and the AChR perimeter ( $p>0.9999)$ and area $(\mathrm{p}>0.9999)$ of SRT were larger than the NT; the endplate compactness $(\mathrm{p}<0.01)$ was even larger. The endplate diameter $(\mathrm{p}=0.1893)$ and the number of AChR clusters of ( $>0.9999)$ SRT were smaller compared to NT. The SRT presented an endplate fragmentation index of 0.51 (Figure 2).

The area $(\mathrm{p}<0.01)$ and perimeter $(\mathrm{p}<0.05)$ of endplate, and the AChR area $(p>0.9999)$ and perimeter $(p<0.01)$ of plantaris were smaller in SRT than that found in the ST. Besides, the AChR area $(\mathrm{p}=0.0303)$, the endplate compactness $(\mathrm{p}<0.05)$, and the number of AChR clusters $(\mathrm{p}<0.01)$ of SRT were larger than ST. The endplate diameter was smaller in SRT than in ST (p=0.0049) (Figure 2).

In SRT, the AChR $(\mathrm{p}<0.01)$ area and perimeter, and the area and perimeter of the endplate $(\mathrm{p}<0.0001)$ were smaller compared to RT. Moreover, the endplate diameter of SRT was smaller than that found in RT $(\mathrm{p}<0.05)$. The endplate compactness and the number of AChR clusters SRT were both larger than RT ( $\mathrm{p}=0.4495)$ (Figure 2). 

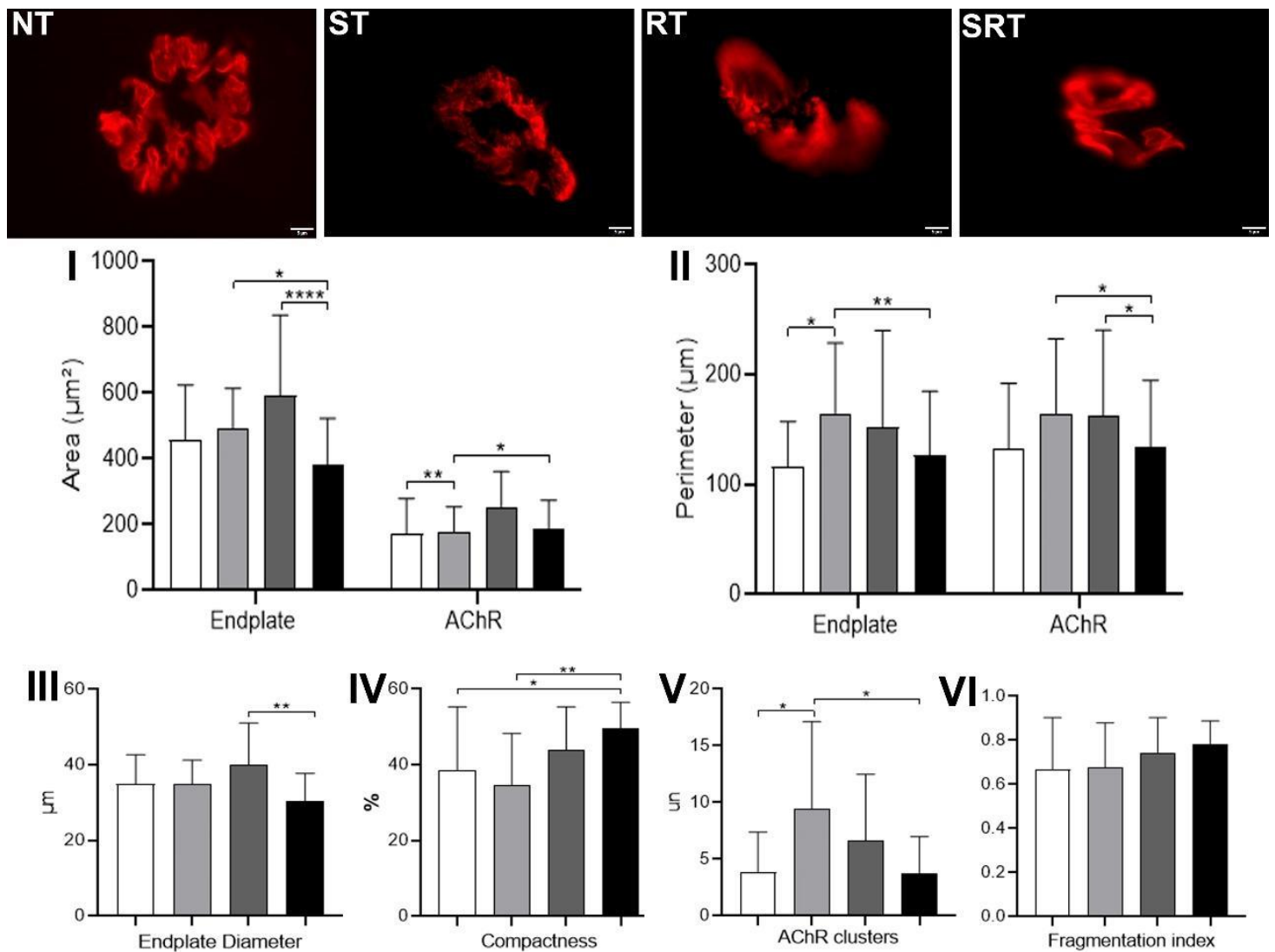

Figure 2: Postsynaptic AChR region of the plantaris muscle of No-Training (NT), Stretching Training (ST), Resistance Training (RT), and Stretching-Resistance Training (SRT) groups. Bars: $5 \mu \mathrm{m}$. (I) Mean \pm standard deviation values of the endplate and AChR areas $\left(\mu \mathrm{m}^{2}\right) * \mathrm{ST} \neq \mathrm{SRT}(\mathrm{p}<0.05) ; * * * * \mathrm{RT} \neq \mathrm{SRT}(\mathrm{p}<0.0001) ; * * \mathrm{NT} \neq \mathrm{ST}(\mathrm{p}<0.01)$; $* \mathrm{ST} \neq \mathrm{SRT}(\mathrm{p}<0.05)$. (II) Mean \pm standard deviation values of the endplate and AChR perimeters $(\mu \mathrm{m}) * \mathrm{NT} \neq \mathrm{ST} \quad(\mathrm{p}<0.05) ; * * \mathrm{ST} \neq \mathrm{SRT} \quad(\mathrm{p}<0.01) ; \quad * \mathrm{ST} \neq \mathrm{SRT} \quad(\mathrm{p}<0.05)$; $* \mathrm{RT} \neq \mathrm{SRT}(\mathrm{p}<0.05)$. (III) Mean \pm standard deviation values of endplate diameter $(\mu \mathrm{m})$ $* * \mathrm{RT} \neq \mathrm{SRT}(\mathrm{p}<0.01)$. (IV) Mean \pm standard deviation values of endplate compactness $(\%) * \mathrm{NT} \neq \mathrm{SRT}(\mathrm{p}<0.05) ;{ }^{*} \mathrm{ST} \neq \mathrm{SRT}(\mathrm{p}<0.05)$. (V) Mean \pm standard deviation values of the number of AChR clusters (un) $* \mathrm{NT} \neq \mathrm{ST}(\mathrm{p}<0.05) ;{ }^{*} \mathrm{ST} \neq \mathrm{SRT}(\mathrm{p}<0.05)$. (VI) Mean \pm standard deviation values of the fragmentation index $* * \mathrm{NT} \neq \mathrm{ST}(\mathrm{p}<0.01) ; * * \mathrm{ST} \neq \mathrm{SRT}$ $(\mathrm{p}<0.01)$.

\section{Discussion}

Evidence suggests an increase in NMJ activity, specifically in the postsynaptic region after resistance training ${ }^{17}$. Our recent finding revealed the effects of static stretching, resistance training, and their association in the myofibers CSA and postsynaptic region. These findings are distinct between the muscles regarding the morphofunctional and myofiber characteristics.

Regular resistance training is the exercise intervention to promote muscular adaptations, such as increases in muscle mass, CSA, myofibrillar protein synthesis, and 
sarcomeres length ${ }^{7,18,19,20}$. In the RT Group, we observed muscle hypertrophy through the increase in CSA of type I of soleus and type IIx of plantaris muscle. These adaptations that occur in the architecture of muscle fibers are directly associated with muscle strength production, where fibers with higher CSA values can develop greater muscle strength production due to the formation of new sarcomeres ${ }^{21,22}$.

Also, Allen et al. ${ }^{23}$ suggested that muscle hypertrophy is associated with the addition of new myonuclei through satellite cells' activity. A recent study by Rocha et al. ${ }^{20}$ showed soleus muscle hypertrophy in both types of fibers after 24 resistance training sessions in a vertical ladder.

Regarding the static stretching response in the CSA of the muscle fibers, Peviani et al. ${ }^{24}$ demonstrated no difference in the soleus of experimental groups after 10 and 15 days of static stretching. In humans, Simpson et al. ${ }^{25}$ observed an increase in the lower limb's muscular thickness after 6 weeks of overloaded stretch training. These adaptations seem to occur according to stretching protocol intensity.

Nunes et al. $^{26}$ indicates that the stretching with external overload does a hypertrophic muscle effect, and changes in muscle size and architecture did not happen in the low-intensity stretch. Our results revealed a reduction in both myofibrillar types compared to the NT group in soleus, differently in plantaris that presented muscle hypertrophy. This fact suggests that this static stretching protocol is intense enough to make beneficial adaptations only in plantaris muscle fibers.

Regular stretching practice can be beneficial in maximum voluntary contraction, and running speed ${ }^{27}$. Our findings reveal that static stretching causes changes in the entire postsynaptic neuromuscular structure, and probably in its function.

Similar to resistance training, manipulating variables in static stretching training seems to play a key role in neuromuscular adaptations. In soleus Type I fibers, when static stretching is combinated with resistance training, we can observe a non-significant increase in CSA. In contrast, the highest CSA value was considered in Type II fibers after previous static stretching to resistance training. Probably, the hypertrophic response induced by stretching before resistance exercise seems to affect fast fibers, presumably due to the stretching of these fibers caused by static stretching and the tension generated through the load additional in resistance training. 
The current literature suggests that low-intensity muscle stretching does not seem to respond significantly to muscle hypertrophy analyzed through CSA measurement; however, high-intensity stretching can cause possible adaptations that are still limited ${ }^{28}$. The practical implication of not performing the previous stretching is a shorter duration of the training sessions, improving adherence to exercise ${ }^{28,29}$.

In this study, changes in the postsynaptic region regarding static stretching and resistance training provided new data on the peripheral nervous system's plasticity. We observed a reduction in the AChR and endplate perimeters, AChR and endplate areas, and consequently, the soleus postsynaptic region's compactness. Indeed, we observed an enlargement of the plantaris endplate and AChR area in ST and RT Group, interrupted when the stretching is performed before the resistance training (SRT Group).

Morphological remodeling of the $\mathrm{AChR}$ area may be associated with desensitization during intense and repeated neural stimulation ${ }^{17}$. This area's increase has a functional consequence of decreasing peripheral muscle fatigue during high-intensity muscle contractions ${ }^{17}$. However, this area's reduction does not imply that the motor neuron's innervation in the muscle fibers is restricted since the postsynaptic potential usually has an amplitude that significantly exceeds the necessary to obtain a postsynaptic action potential ${ }^{30}$.

Although evidence suggests that stretching before exercise decreases the results of performance tests that require muscle strength or power ${ }^{27}$, data on stretching in other perspectives are limited, mainly its influence on the postsynaptic responses.

Regarding resistance training on a vertical ladder, Deschenes et al. ${ }^{31}$ presented data on young and elderly animals, where there were adaptations only in the postsynaptic components of the NMJ, as is evident in our RT data. The use of load in training sessions on a vertical ladder results in the morphological increase of the NMJ of adult rat's soleus ${ }^{17}$.

Resistance training after static stretching (SRT Group) resulted in higher endplate compactness than other experimental groups. The term compactness has been used as a descriptor of quantity receptors in the postsynaptic area ${ }^{32}$; in other studies, we can find this index as a "dispersion" ${ }^{33,34}$. Deschenes et al. ${ }^{3}$ documented that compactness (or dispersion) is directly related to the intensity of exercise in which the resistance training increased soleus postsynaptic compactness, and in low-intensity running, 
reduced endplate dispersion ${ }^{17}$. The resistance training after stretching requires high neuromuscular activity, whereas the alterations in the distribution of synaptic receptors within the endplate region are induced by exercise type ${ }^{35}$. We can verify that the training intensity can influence the alterations in the receptors in the postsynaptic region.

The endplate diameter showed changes similar to the endplate area, which are minor values in stretching groups. In Boehm et al. ${ }^{36}$ work, each animal species has a different value of endplate diameter. Indeed, Estrada-Bonilla et al. ${ }^{37}$ observed the NMJ morphometric characteristics of the upper limbs of healthy and diabetes mellitusinduced rats. These data differ from the lower limbs found in this study. However, there were also minor values in the diameter in conditions of diabetes compared to the control $^{37}$.

About the fragmentation index, Prakash and Sieck ${ }^{38}$ found the effects of aging on morphological adaptations of NMJs such as altered fragmentation. Although fragmentation may result from the redistribution of pre-synaptic components over a greater synaptic area, these alterations can also represent an increase in denervation and reinnervation, which may compromise motor innervation and contribute to muscle weakness $^{21}$. As a result, these exercise-induced alterations need to be considered in training protocols or rehabilitation exercises to improve neuromuscular function ${ }^{35}$.

\section{Conclusion}

In conclusion, both groups submitted to resistance training showed similar muscle hypertrophy results, highlighting no previous static stretching. The musclespecific adaptation in the postsynaptic region occurs according to the training, in which the resistance training showed greater morphological remodeling. However, the previous static stretching changes this remodeling.

\section{Materials and Methods}

\section{Animals}

Thirty-two male Wistar rats with 60 days-old were divided into 4 Groups ( $\mathrm{n}=$ 8): No-Training (NT); Resistance Training (RT); Stretching Training (ST); and Stretching-Resistance Training (SRT). During the experimental period, the rats were allocated in cages $(33 \times 40 \times 16 \mathrm{~cm})(\mathrm{n}=4)$, under the conditions of temperature monitoring $\left(23 \pm 2{ }^{\circ} \mathrm{C}\right)$ and $12 \mathrm{~h}$ light/dark period, with food and water "ad libitum". All 
proceedings were approved by the Animal Use Ethics Committee (CEUA)-UNESP (No. 09/2019) and carried out following the National Council for Animal Experiment Control (CONCEA). This study was carried out in compliance with the ARRIVE guidelines.

\section{Stretching Training}

The ST and SRT Groups performed an 8-week (24 sessions) stretching protocol (3x/week), which consisted of using the non-quantified manual force applied to the plantar portion of the right posterior limb with the movement of dorsiflexion up to the range of motion limit of the talocrural joint. With the manual assistance of a specific investigator, the rats performed 10 movements of 30 seconds of static stretching, followed by a 30 -second interval resting in the neutral joint position ${ }^{39}$. The same investigator executed all sessions. SRT Group performed the stretching and immediately followed by resistance training in a vertical ladder.

\section{Resistance Training Protocol}

The rats of RT and SRT Groups performed an 8-week (24 sessions) resistance training protocol (3x/week) in a vertical ladder $\left(110 \mathrm{x} 18 \mathrm{~cm}, 2 \mathrm{~cm}\right.$ grid, $80^{\circ}$ incline $)$. The sessions consisted of 4 to 9 progressive load climbs. They were allowed to rest for 120 seconds at the top of the ladder after each climb $^{40}$.

The rats performed the first four climbs with 50\%, 75\%, 90\%, and $100 \%$ of their body mass additional loads fixed to the tail's proximal region ${ }^{41}$. In the subsequent climbs, $30 \mathrm{~g}$ of the extra progressive load was added until the ninth climb or exhaustion occurred $^{18}$. The rats performed the training protocols at the same time of the day across the experimental period.

\section{Histochemistry}

The belly samples of soleus and plantaris muscle ( $n=5$ in each) of experimental groups were dissected and cryofixed, then transverse sections were made $(10 \mu \mathrm{m}$ thickness) (Cryostat HM 505 E, MICROM ${ }^{\mathrm{TM}}$ ). The histochemical reaction was used for myosin adenosine triphosphatase (ATPase) and the $\mathrm{pH}$ lability to differentiate the fiber types (Type I and II in soleus/ Type I, IIa, and IIx in plantaris muscle) ${ }^{20}$.

For $\mathrm{pH} 4.3$ and 4.6 , the sections were pre-incubated in $0.1 \mathrm{M}$ sodium acetate buffer solution and $10 \mathrm{mM}$ ethylenediaminetetraacetic acid (EDTA) solution for $10 \mathrm{~min}$ at $4{ }^{\circ} \mathrm{C}$. All the sections were incubated in a solution containing ATP (10 mg), 
glycine/ $\mathrm{NaCl}$ buffer $\left(10 \mathrm{~mL}\right.$ ), $\mathrm{CaCl}_{2}$ (in $\mathrm{pH} 9.4$ ), and dithiothreitol for $30 \mathrm{~min}$. The sections were incubated in $2 \%$ cobalt chloride for $7 \mathrm{~min}$, dehydrated in a series of alcohol concentrations, and finished in xylol ${ }^{33}$.

\section{Morphologic and morphometric analysis}

The images were obtained by a Zeiss ${ }^{\text {TM }}$ Axioskop (Jena, Germany) light microscope. After, the morphometry was performed in each $\mathrm{pH}$ by the ImageJTM software. The cross-section area (CSA) of Type I and II of soleus myofibers and the CSA of Type I, IIa, and IIx of plantaris myofibers ( $n=100 /$ fiber type/group) were quantified using a 20X objective lens with 10X ocular magnification. Subsequently, we performed the normality test of the data and analyzed it using Kruskal-Wallis with Dunn's post hoc test $(\mathrm{p}<0.05)^{33}$.

\section{Postsynaptic imaging}

\section{Tissue preparation}

The samples of soleus and plantaris muscle $(n=3$ in each) of experimental groups were dissected and cryo fixed in liquid nitrogen $\left(-196^{\circ} \mathrm{C}\right)$, then longitudinal sections (100 $\mu \mathrm{m}$ thickness) were made (Cryostat HM 505 E, MICROM ${ }^{\mathrm{TM}}$ ) to visualize the postsynaptic component of each NMJ. The sections were collected in silanized slides, pre-treated with $0.1 \%$ Triton-X solution, and washed $(3 \times 5 \mathrm{~min})$ in phosphatebuffered saline (PBS). The sections were incubated overnight at $4{ }^{\circ} \mathrm{C}$ in a solution containing $\alpha$-bungarotoxin conjugated with rhodamine (BTX; Molecular Probes ${ }^{\mathrm{TM}}$, Eugene, OR-T-1175), diluted 1:600 in PBS containing 1\% bovine serum albumin (BSA); washed in PBS before being coated with Prolong (Molecular Probes ${ }^{\mathrm{TM}}$, Eugene, OR- P10144), coverslips applied and stored at $-20{ }^{\circ} \mathrm{C}$ until analysis ${ }^{33}$.

\section{Morphometric analysis}

The images $(\mathrm{n}=20)$ for morphometric analysis were captured using a $100 \mathrm{X}$ objective lens with 10X ocular magnification by an Olympus BX61 ${ }^{\mathrm{TM}}$ Fully Motorized Fluorescence Microscope (Shinjuku, Japan); equipped with a Fluorescence UIS2 optical system by TexasRed filter (Texas red excitation $596 \mathrm{~nm} / \mathrm{emission} 620 \mathrm{~nm}$ ), obtained by a monochromatic camera Orca-Flash 2.8 (Hamamatsu, Japan) with the Software CellSens v.11 (Olympus ${ }^{\mathrm{TM}}$ ). 
With the use of the Image ${ }^{\mathrm{TM}}$ Software, it was measured the endplate area $\left(\mu \mathrm{m}^{2}\right)$ : the entire region composed of stained and non-stained receptors; AChR area $\left(\mu \mathrm{m}^{2}\right)$ : the area composed of AChR; endplate perimeter $(\mu \mathrm{m})$ : the whole length covering the endplate composed of stained and non-stained receptor; AChR perimeter $(\mu \mathrm{m})$ : the size covering around the region composed of AChR; endplate diameter $(\mu \mathrm{m})$ : the maximum diameter formed by stained and non-stained receptors; endplate compactness (\%): quantity of receptors in the postsynaptic area (AChR area/endplate area) x 100, in other studies we can found this index as a "dispersion" 33,34; the number of AChR clusters (un): the number of $\mathrm{AChR}$ clusters comprising the motor endplate; and endplate fragmentation index: 1-(1/number of AChR clusters), whereby an index of 0 means a solid plaque-like endplate, and an index close to 1 means a highly fragmented endplate $^{32}$. We performed the normality test of the data and analyzed using KruskalWallis with Dunn's post hoc test $(p<0.05)$.

Data availability: All relevant data are within the paper.

\section{References}

${ }^{1}$ Deschenes, M. R. Adaptations of the neuromuscular junction to exercise training. Curr. Opin. Physiol. 10, 10-16 (2019)

${ }^{2}$ Deschenes, M. R. et al. Muscle fibers and their synapses differentially adapt to aging and endurance training. Exp. Gerontol. 106, 183-191 (2018)

${ }^{3}$ Deschenes, M. R. et al. The effects of exercise training of different intensities on neuromuscular junction morphology. J. Neurocytol. 22, 603-615 (1993)

${ }^{4}$ Fahim, M. A. Endurance exercise modulates neuromuscular junction of C57BL/6NNia aging mice. J. Appl. Physiol. 83, 59-66 (1997)

${ }^{5}$ Baehr, L. M. et al. Age-related deficits in skeletal muscle recovery following disuse are associated with neuromuscular junction instability and ER stress, not impaired protein synthesis. Aging 8, 127-146 (2016)

${ }^{6}$ Kraemer, W. J., Ratamess, N. A. Fundamentals of Resistance Training: Progression and Exercise Prescription. Med. Sci. Sports Exerc. 36, 674-688 (2004)

${ }^{7}$ Schoenfeld, B. et al. Effects of different volume-equated resistance training loading strategies on muscular adaptations in well-trained men. J. Strength Cond. Res. 28, 2909-2918 (2014)

${ }^{8}$ Marzetti, E. et al. Physical activity and exercise as countermeasures to physical frailty and sarcopenia. Aging Clin. Exp. Res. 29, 35-42 (2017)

${ }^{9}$ Qaisar, R., Bhaskaran, S., Van Remmen, H. Muscle fiber type diversification during exercise and regeneration. Free Radic. Biol. Med. 98, 56-67 (2016) 
${ }^{10}$ Sá, M. A. et al. Acute effects of different methods of stretching and specific warmups on muscle architecture and strength performance. J. Strength Cond. Res. 30, 2324-2329 (2015)

${ }^{11}$ Kamonseki, D. H., Gonçalves, G. A., Yi, L. C., Lombardi Júnior, I. Effect of stretching with and without muscle strengthening exercises for the foot and hip in patients with plantar fasciitis: A randomized controlled single-blind clinical trial. Man. Ther. 23, 76-82 (2014)

${ }^{12}$ Jamtvedt, G. et al. (2010) A pragmatic randomised trial of stretching before and after physical activity to prevent injury and soreness. Br. J. Sports Med. 44, 1002 1009 (2010).

${ }^{13}$ Palmer, T. B., Agu-Udemba, C. C., Palmer, B. M. Acute effects of static stretching on passive stiffness and postural balance in healthy, elderly men. Phys. Sportsmed. 46, 78-86 (2018)

${ }^{14}$ Leite, T. et al. Influence of strength and flexibility training, combined or isolated, on strength and flexibility gains. J. Strength Cond. Res. 29, 1083-1088 (2015)

${ }^{15}$ Barroso, R., Tricoli, V., Gil, S. S., Ugrinowitsch, C., Roschel, H. Maximal strength, number of repetitions, and total volume are differently affected by static-, ballistic-, and proprioceptive neuromuscular facilitation stretching. J. Strength Cond. Res. 26, 2432-2437 (2012)

${ }^{16}$ Moriggi Júnior, R., Berton, R., de Souza, T. M. F., Chacon-Mikahil, M. P. T., Cavaglieri, C. R. Effect of the flexibility training performed immediately before resistance training on muscle hypertrophy, maximum strength and flexibility. Eur. J. Appl. Physiol. 117, 767-774 (2017)

${ }^{17}$ Deschenes, M. R. et al. Effects of resistance training on neuromuscular junction morphology. Muscle Nerve 23, 1576-1581 (2000)

${ }^{18}$ Pimentel Neto, J. et al. Myotendinous junction adaptations to ladder-based resistance training: identification of a new telocyte niche. Sci. Rep. 10, 14124 (2020)

${ }^{19}$ Burd, N. A. et al. Resistance exercise volume affects myofibrillar protein synthesis and anabolic signalling molecule phosphorylation in young men. J. Physiol. 588, 3119-3130 (2010)

${ }^{20}$ Rocha, L. C. et al. Repercussions on sarcomeres of the myotendinous junction and the myofibrillar type adaptations in response to different trainings on vertical ladder. Microsc. Res. Tech. 83, 1190-1197 (2020a)

${ }^{21}$ Zamparo, P., Minetti, A. E., Di Prampero, P. E. Interplay among the changes of muscle strength, cross-sectional area and maximal explosive power: Theory and facts. Eur. J. Appl. Physiol. 88, 193-202 (2002)

${ }^{22}$ Suchomel, T. J., Nimphius, S., Bellon, C. R., Stone, M. H. The Importance of Muscular Strength: Training Considerations. Sport Med. 48, 765-785 (2018)

${ }^{23}$ Allen, D. L., Roy, R. R. \& Edgerton, V. R. Myonuclear domains in muscle adaptation and disease. Muscle Nerve 22, 1350-1360 (1999)

${ }^{24}$ Peviani, S. M. et al. Regulation of extracellular matrix elements and sarcomerogenesis in response to different periods of passive stretching in the soleus 
muscle of rats. Sci. Rep. 8, 9010 (2018)

${ }^{25}$ Simpson CL, Kim BDH, Bourcet MR et al Stretch training induces unequal adaptation in muscle fascicles and thickness in medial and lateral gastrocnemii. Scand. J. Med. Sci. Sport 27, 1597-1604 (2017)

${ }^{26}$ Nunes, J. P. et al. Does stretch training induce muscle hypertrophy in humans? A review of the literature. Clin. Physiol. Funct. Imaging 40, 148-156 (2020)

${ }^{27}$ Shrier, I. Does stretching improve performance? A systematic and critical review of the literature. Clin. J. Sport Med. 14, 267-273 (2004)

${ }^{28}$ Nuzzo, J. L. The case for retiring flexibility as a major component of physical fitness. Sport Med. 50, 1-13 (2019)

${ }^{29}$ Hoare, E., Stavreski, B., Jennings, G. L., Kingwell, B. A. Exploring motivation and barriers to physical activity among active and inactive australian adults. Sports (Basel) 5, 1-8 (2017)

${ }^{30}$ Finsterer, J. Biomarkers of peripheral muscle fatigue during exercise. BMC Musculoskelet. Disord. 13, 1-13 (2012)

${ }^{31}$ Deschenes, M. R., Sherman, E. G., Roby, M. A., Glass, E. K., Harris, M. B. Effects of resistance training on neuromuscular junctions of Young and aged muscles featuring recruitment patterns. J. Neurosci. Res. 93, 504-513 (2015).

${ }^{32}$ Jones, R. A. et al. NMJ-morph reveals principal components of synaptic morphology influencing structure-function relationships at the neuromuscular junction. Open Biol. 6, 1-16 (2016)

${ }^{33}$ Rocha, L. C. et al. Remodeling of the skeletal muscle and postsynaptic component after short-term joint immobilization and aquatic training. Histochem. Cell Biol. 154, 621-628 (2020b)

${ }^{34}$ Deschenes, M. R., Hurst, T. E., Ramser, A. E., Sherman, E. G. Presynaptic to postsynaptic relationships of the neuromuscular junction are held constant across age and muscle fiber type. Dev. Neurobiol. 73, 744-53 (2013)

${ }^{35}$ Deschenes, M. R. et al. Effects of exercise training on neuromuscular junctions and their active zones in young and aged muscles. Neurobiol. Aging. 95, 1-8 (2020)

${ }^{36}$ Boehm, I. et al. Comparative anatomy of the mammalian neuromuscular junction. J. Anat. 237, 827-836 (2020)

${ }^{37}$ Estrada-Bonilla, Y. C. et al. Reaching task performance is associated to neuromuscular junction adaptations in rats with induced diabetes mellitus. Braz. J. Med. Biol. Res. 53, e8763 (2020)

${ }^{38}$ Prakash, Y. S., Sieck, G. C. Age-related remodeling of neuromuscular junctions on type-identified diaphragm fibers. Muscle Nerve 21, 887-895 (1998)

${ }^{39}$ Gianelo, M. C. S., Polizzelo, J. C., Chesca, D., Mattiello-Sverzut, A. C. Three days of intermittent stretching after muscle disuse alters the proteins involved in force transmission in muscle fibers in weanling rats. Braz. J. Med. Biol. Res. 49, 1-8 (2016)

${ }^{40}$ Hornberger, T. A., Farrar, R. P. Physiological hypertrophy of the FHL muscle 
following 8 weeks of progressive resistance exercise in the rat. Can. J. Appl. Physiol. 29, 16-31 (2004)

${ }^{41}$ Krause Neto, W., Silva, W. A., Ciena, A. P., Anaruma, C. A., Gama, E. F. Divergent effects of resistance training and anabolic steroid on the postsynaptic region of different skeletal muscles of aged rats. Exp. Gerontol. 98, 80-90 (2017)

${ }^{42}$ Brooke, M. H., Kaiser, K. K. Muscle Fiber Types: How Many and What Kind? Arch. Neurol. 23, 369-379 (1970)

Acknowledgments: We thank Professor Henrique Ferreira for the support with Fluorescence Microscope.

Author's contributions: A.P.C., C.S.J. and G.K.B. conceived and designed the research study; C.S.J., G.K.B., and M.R.P. performed the training protocols; A.P.C., C.S.J., G.K.B., J.P.N., and L.C.R. analyzed data; C.S.J. and A.P.C. wrote the manuscript. All authors read and approved the final manuscript.

Funding: This study was financed the São Paulo Research Foundation (Process: 2017/12525-1), and Coordenação de Aperfeiçoamento de Pessoal de Nível SuperiorBrasil (CAPES) - Finance Code 001.

Conflicts of interest: The authors declare no conflicts of interests. 

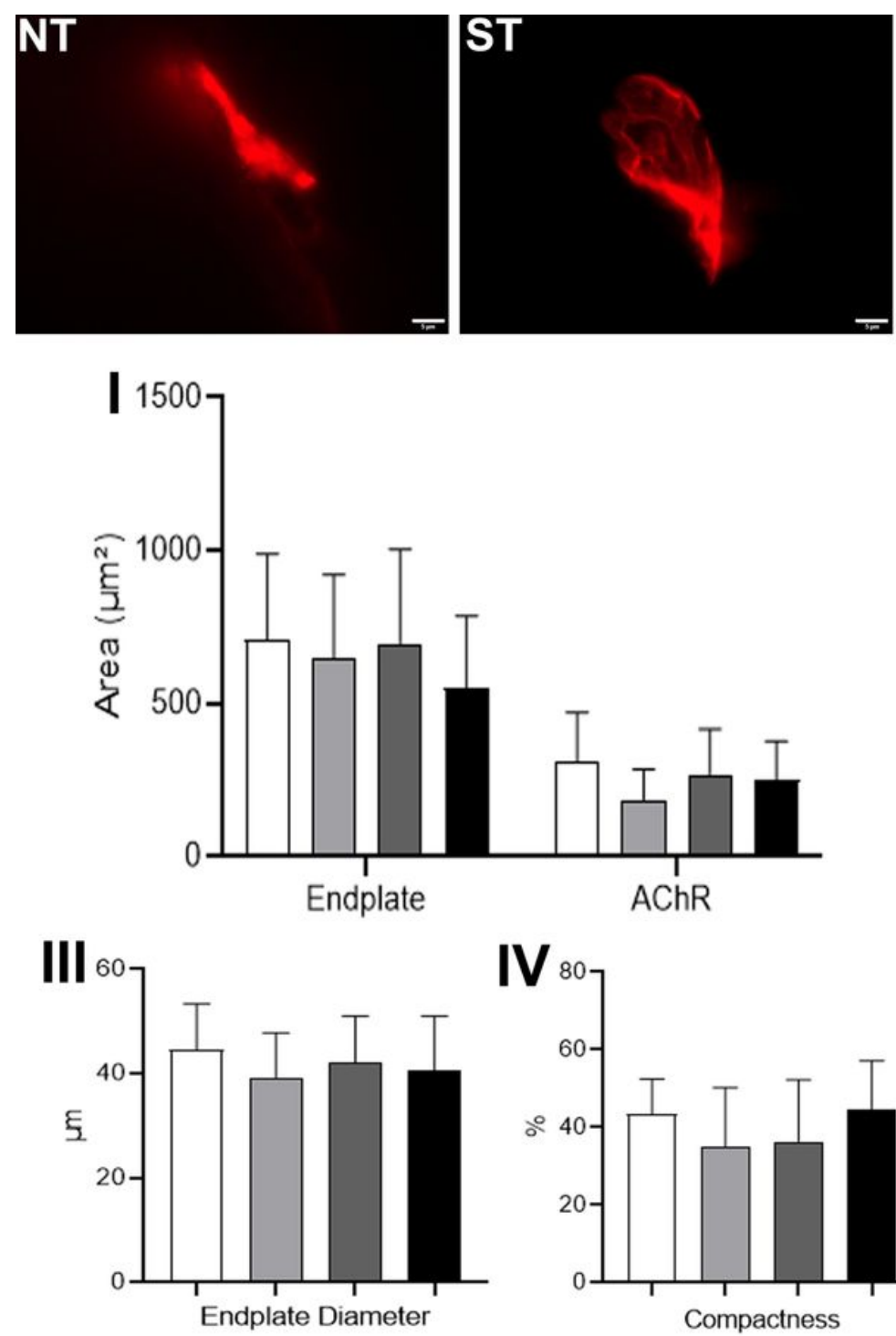
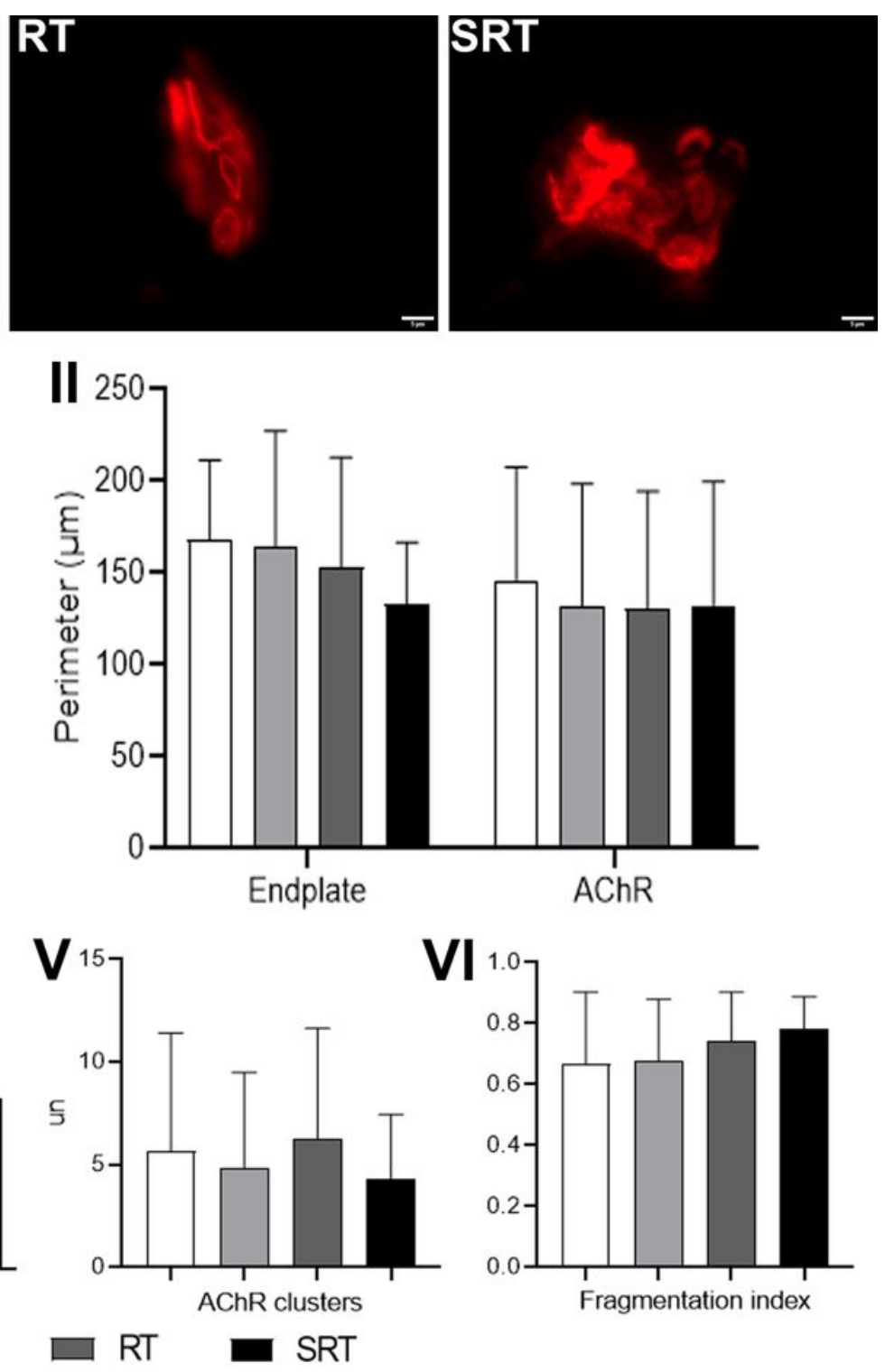

Figure 1

Postsynaptic AChR region of the soleus muscle of No-Training (NT), Stretching Training (ST), Resistance Training (RT), and Stretching-Resistance Training (SRT) groups. Bars: $5 \mu \mathrm{m}$. (I) Mean \pm standard deviation values of the endplate and $A C h R$ areas $\left(\mu \mathrm{m}^{2}\right)$. (II) Mean \pm standard deviation values of the endplate and AChR perimeters $(\mu \mathrm{m})$. (III) Mean \pm standard deviation values of endplate diameter $(\mu \mathrm{m})$. (IV) Mean \pm standard deviation values of endplate compactness (\%). (V) Mean \pm standard deviation values of the number of AchR clusters (un). (VI) Mean \pm standard deviation values of the fragmentation index. 

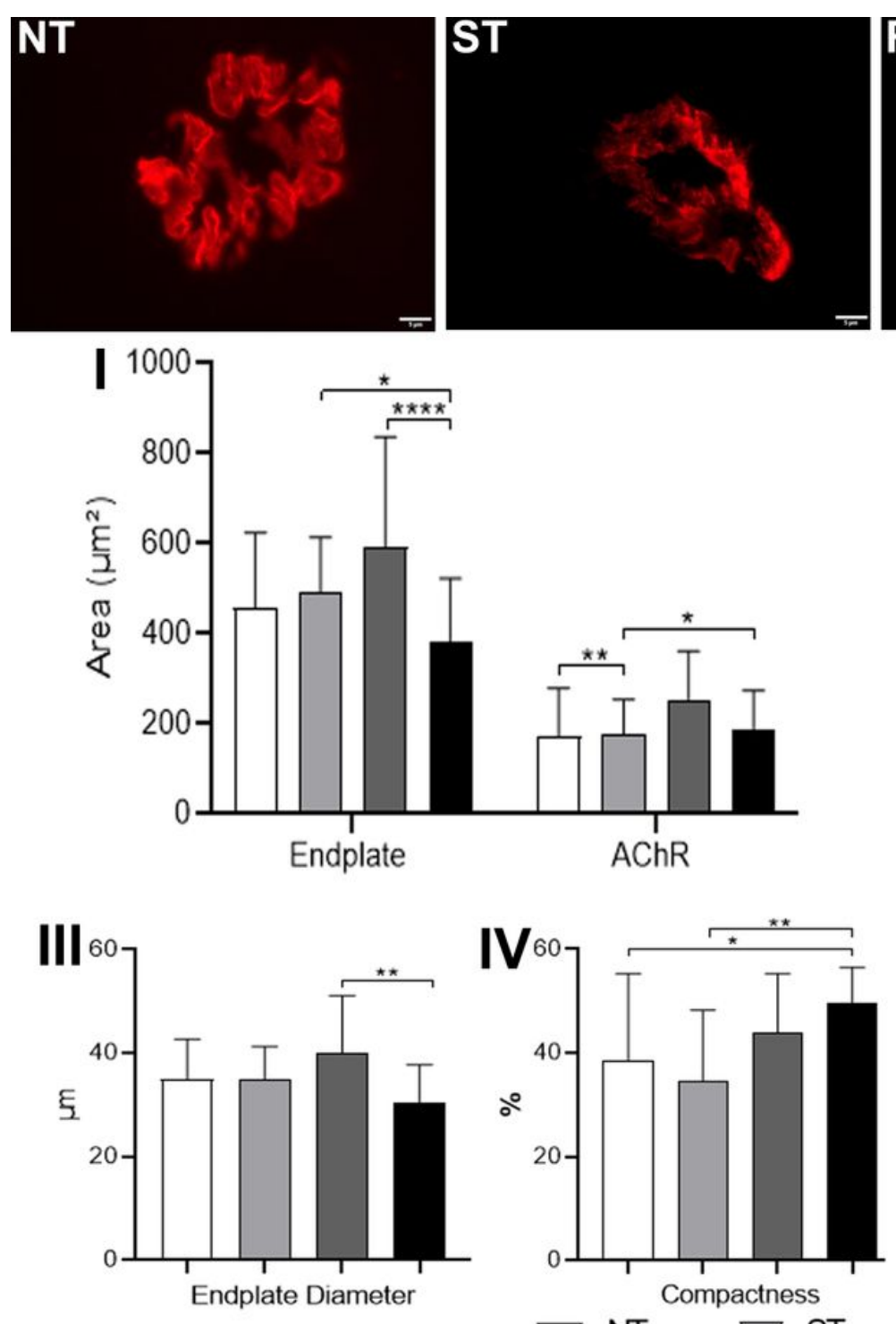

NT

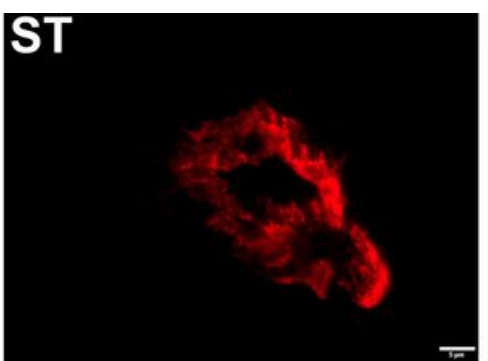

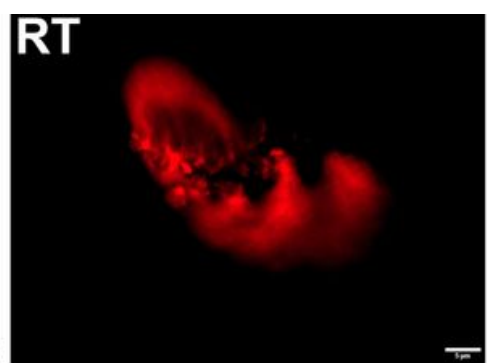
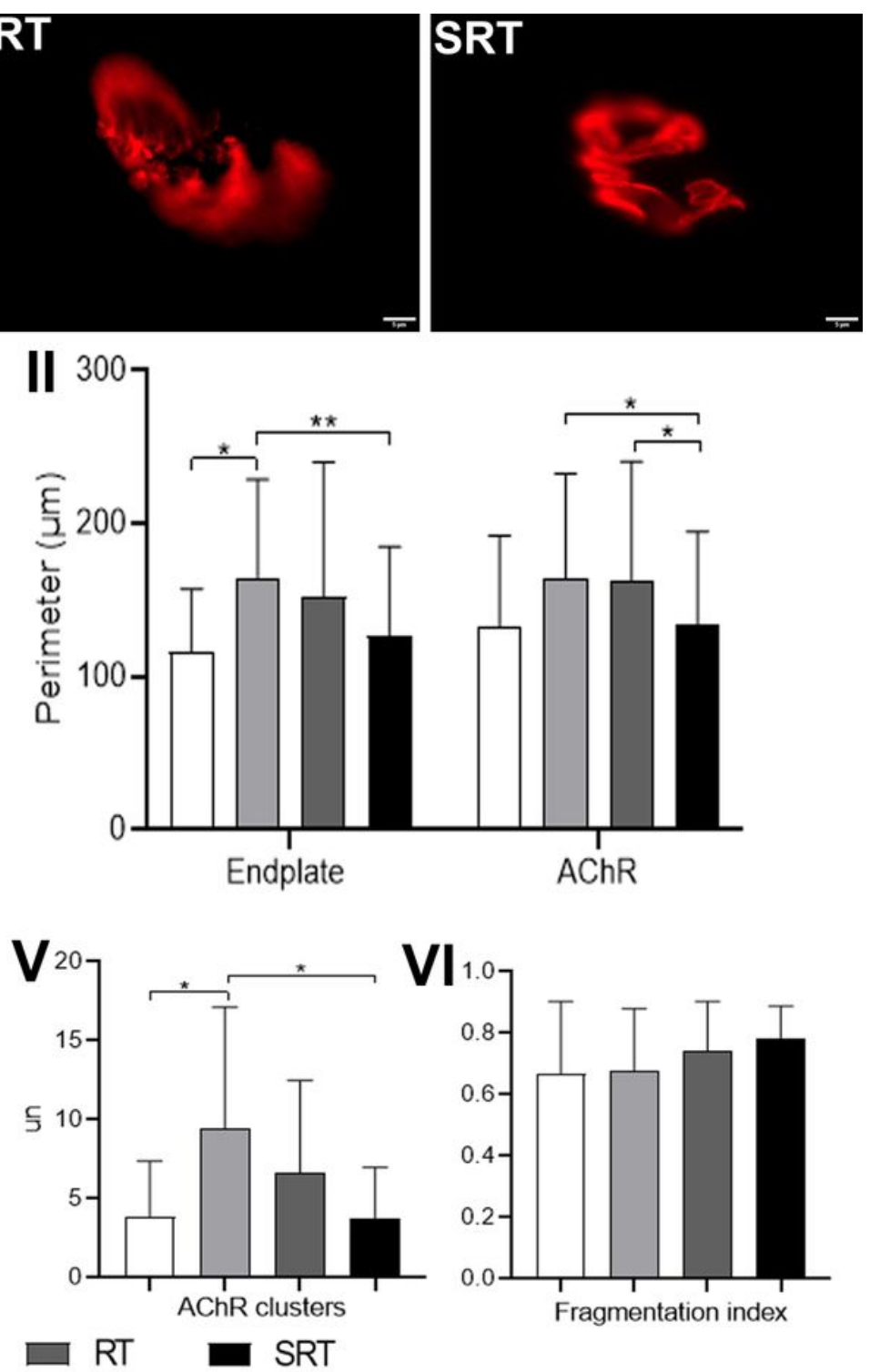

Figure 2

Postsynaptic AChR region of the plantaris muscle of No-Training (NT), Stretching Training (ST), Resistance Training (RT), and Stretching-Resistance Training (SRT) groups. Bars: $5 \mu \mathrm{m}$. (I) Mean \pm standard deviation values of the endplate and AChR areas $\left(\mu m^{2}\right) * S T \neq S R T(p<0.05) ; * \star * * R T \neq S R T$ $(p<0.0001) ; * \star N T \neq S T(p<0.01) ; * S T \neq S R T(p<0.05)$. (II) Mean \pm standard deviation values of the endplate and AChR perimeters $(\mu \mathrm{m}) * \mathrm{NT} \neq \mathrm{ST}(\mathrm{p}<0.05) ; * \star S T \neq S R T(p<0.01) ; * S T \neq S R T(p<0.05) ; * R T \neq S R T(p<0.05)$. (III) Mean \pm standard deviation values of endplate diameter $(\mu \mathrm{m}){ }^{*} \mathrm{RT} \neq \mathrm{SRT}(\mathrm{p}<0.01)$. (IV) Mean \pm standard deviation values of endplate compactness $(\%) * N T \neq S R T(p<0.05)$; ${ }^{*} S T \neq S R T(p<0.05)$. (V) Mean

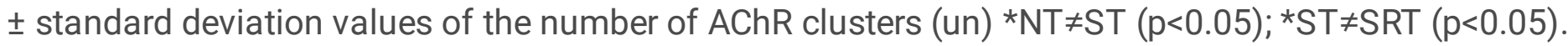

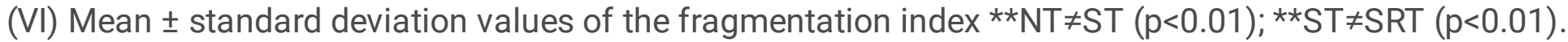

